\title{
Concevoir la complexité temporelle en éducation dans une perspective critique
}

\section{Michel Alhadeff-Jones}

L'étude du temps et des rythmes de l'éducation révèle une complexité particulièrement difficile à analyser dans la mesure où elle renvoie simultanément à des registres hétérogènes de l'expérience (p.ex., physique, biologique, psychologique, social, culturel). Cet article propose de l'appréhender en revisitant certains des présupposés à partir desquels les temporalités de l'éducation et de la formation sont envisagées. Cette réflexion s'organise autour de trois axes: le premier vise à rendre compte de l'hétérogénéité des temporalités éducationnelles, le deuxième explore leur nature dynamique et conflictuelle et le troisième axe interroge plus spécifiquement la portée critique d'une réflexion sur la complexité temporelle, dans le cadre d'une éducation à visée émancipatrice.

\section{Reconnaître la complexité temporelle en éducation 1}

Le temps représente à la fois un concept crucial pour interpréter l'expérience vécue et une dimension implicite de la vie quotidienne. Il est inhérent aux aspects les plus profonds de l'existence (notre propre finitude), en même temps qu'il permet de réguler les routines quotidiennes les plus superficielles. Il peut être conçu comme étant universel (p.ex., les saisons), tout en permettant de disposer d'une ressource pour mesurer des changements particuliers (p.ex., au sein d'une organisation); son appréciation demeure toutefois contingente, dans la mesure où elle dépend toujours de facteurs historiques, géographiques, psychologiques, sociaux et culturels (Adam, 1994; Chenet, 2000; Dubar, 2008; Fraisse, 1957; Grossin, 1996). Ainsi, il est collectivement défini (p.ex., calendriers, âges de la vie), mais sa perception varie en fonction des expériences personnelles vécues (p.ex., notre humeur, notre histoire de vie). Quand elle permet la planification et la synchronisation d'opérations multiples, la référence au temps offre un sentiment de cohérence et de prévisibilité qui constituent une source d'ordre; mais quand elle est vécue à travers des demandes temporelles conflic- 
tuelles (p.ex., au travail, en famille), elle apparait comme une source de stress, de confusion, et de souffrance qui déstabilisent. L'idée de temps est souvent considérée comme neutre, toutefois les significations qu'elle prend (p.ex., être rapide/lent, en avance/en retard, mature/immature) demeurent toujours socialement construites. Et parce que ces significations déterminent le déroulement des actions entreprises, le rapport au temps s'inscrit fondamentalement dans des rapports de forces. Si l'idée de temps suggère une forme de singularité, les notions de temporalité et de rythme permettent d'en penser la pluralité des expressions (Adam, 1994; Dubar, 2008; Michon, 2005). Les multiples formes de changement auxquelles celles-ci réferent (p.ex., individuel, organisationnel, institutionnel, environnemental), contribuent toutes à la définition de ce que nous sommes, à la manière dont on se comporte et dont on apprend. L'éducation apparaît dès lors façonnée par des temporalités hétérogènes, à la fois complémentaires, antagonistes et contradictoires, qui rythment l'activité, la vie et le développement des apprenants, des éducateurs, des institutions, de la société et des connaissances elles-mêmes. L'éducation détermine également la manière dont on développe un certain rapport au temps et à la pluralité des rythmes de l'existence (p.ex., à travers l'exercice d'une discipline temporelle à l'école, ou à travers les routines familiales). Qu'il soit appréhendé à travers l'expérience quotidienne, le questionnement philosophique, l'expression artistique, les significations religieuses ou les théories scientifiques (Adam, 1994; Chenet, 2000; Dubar, 2008; Fraisse, 1957; Fraser, 1966; Gonord, 2001; Koukkari \& Sothern, 2006; Lurçat, 1995), le temps ne constitue dès lors ni une idée simple qu'il suffirait d'expliquer, ni un phénomène compliqué qu'il s'agirait de décomposer et d'analyser. Le temps devrait plutôt être envisagé comme un phénomène fondamentalement complexe (Alhadeff-Jones, 2017). En tant que tel, il renvoie à des formes hétérogènes de changement qui se produisent à différents "niveaux» de l'existence (p.ex., physique, biologique, psychologique, sociale, esthétique, ou spirituelle). Parce que ces changements sont reliés et enchevêtrés, ils révèlent une complexité particulièrement difficile à saisir et à analyser. Comment dès lors la restituer et la rendre intelligible? Afin d'envisager ce questionnement dans une perspective critique (Alhadeff-Jones, 2007, 2010), la réflexion proposée dans cet article s'organise autour de trois axes: le premier vise à rendre compte de l'hétérogénéité des temporalités éducationnelles, le deuxième explore leur nature dynamique et conflictuelle et le troisième axe interroge plus spécifiquement la portée critique d'une réflexion sur la complexité temporelle, dans le cadre d'une éducation à visée émancipatrice. 


\section{Premier axe de réflexion: reconnâ̂tre l'hétéro- généité des temporalités éducationnelles}

Lorsqu'on prend en considération les aspects temporels de l'éducation, il convient d'emblée de distinguer le traitement implicite et explicite du temps dans la recherche. Parce que l'éducation traite de changements et que leur étude implique une dimension temporelle, le temps constitue une problématique impossible à ignorer. La plupart des recherches en éducation part ainsi du principe que le temps est un facteur externe qui contraint l'apprentissage et le développement, ou qui permet aux chercheurs et aux praticiens de disposer d'un cadre pour décrire la manière dont les processus éducatifs évoluent (Slattery, 1995). De telles hypothèses reposent sur une conception du temps qui ne constitue pas en soi l'objet de la démarche de recherche. Ainsi la plupart des travaux en éducation considère le temps comme une dimension qui ne requiert pas d'être systématiquement questionnée. Lorsqu'on prend au contraire le temps comme objet de réflexion systématique (Alhadeff-Jones, 2017; Ben-Peretz \& Bromme, 1990; Cavet, 2011; Chopin, 2010; Delhaxhe, 1997; Pineau, 2000; St-Jarre \& Dupuy-Walker, 2001), il est pertinent d'établir une seconde distinction entre, d'une part, la recherche sur le temps considéré comme une condition - voire un produit - des processus éducationnels étudiés, et d'autre part, la recherche sur le temps comme objet même de l'apprentissage ou de la formation (Lesourd, 2006). En suivant la démarche proposée par Pomian (1984) en philosophie de l'histoire, on peut distinguer au moins quatre conceptions à travers lesquelles l'idée de temps peut être envisagée en éducation.

\section{Approches chronométriques}

La majorité des recherches en éducation repose sur une approche chronométrique du temps. Le temps est représenté à travers le recours à des calendriers ou à des instruments de mesure. Une telle conception repose sur la possibilité de décrire un cycle invariant qui se répète (p.ex., 24 heures, semaines, mois, ou saisons de l'année). Le temps sert ainsi de structure externe permettant de décrire les moments où des changements se produisent. On retrouve une telle conception dans les recherches conduites autour du «temps de l'enseignement» (instructional time ou academic learning time). Dans cette perspective, la référence au temps permet de disposer d'une mesure quantifiable pour définir et évaluer la plupart des variables du processus d'apprentissage et du dispositif éducatif (Chopin, 2010; Delhaxhe, 1997). De la même manière, la recherche sur les rythmes scolaires (Cavet, 2011), ancrée dans les travaux en chronobiologie et en chronopsychologie (Testu, 2008), permet de disposer d'un cadre pour décrire les changements physiologiques et cognitifs vécus par les apprenants, en les considérant comme quantifiables et mesurables. Lorsque l'on considère le temps comme objet de formation une approche chronométrique conduit à envisager le développement de compétences telles que celles impliquées dans la manière dont 
on "gère» son temps (Larsson \& Sanne, 2005). Une conception chronométrique du temps apparaît comme utile dans la mesure où elle permet de disposer d'une base objective pour mesurer, synchroniser ou planifier les activités observées ou entreprises. Elle demeure toutefois limitée lorsqu'il s'agit d'interpréter des comportements humains manifestant des variations ou des discontinuités dont la quantification est peu pertinente. Elle exclut également de prendre en considération l'expérience intime du temps ou les effets inhérents aux représentations du passé, du présent, ou du futur sur le déroulement de l'activité (Ardoino, 2000; Pineau, 2000; Slattery, 1995).

\section{Approches chronographiques}

Une approche chronographique du temps se centre sur ses attributs qualitatifs. On la retrouve par exemple dans l'écriture de chroniques basées sur l'enregistrement d'événements vécus au quotidien considérés soit comme anormaux, significatifs, surprenants ou extraordinaires, et laissant de côtés les faits qui se répètent de manière redondante (Pomian, 1984). En éducation, on retrouve une telle conception du temps dans les approches qui privilégient la rédaction d'un journal d'apprentissage, utilisé soit pour rendre compte des processus de formation vécus, soit pour promouvoir une activité réflexive. Une conception chronographique se retrouve également lorsqu'on a recours à des processus narratifs. Elle suppose une conception du temps envisagé à la fois comme continu et discontinu. Elle permet de rendre compte de l'histoire d'une personne, d'un collectif ou d'une institution (Pomian, 1984, pp. iv-v). Ainsi, le travail de mise en récit renvoie à différentes méthodologies de recherche (p.ex., approches narratives, biographiques, autoethnographiques) utilisées en éducation pour rendre compte de la manière dont on fait l'expérience de processus d'apprentissage ou de formation. Dans cette optique, l'expérience des temporalités éducationnelles est envisagée comme une construction qu'il s'agit d'interpréter. On retrouve également ce type de conception temporelle au sein d'approches privilégiant la mise en récit comme processus de formation, dans le cadre scolaire (Greene, 1975, citée in Mayes, 2005) ou en formation d'adultes (Dominicé, 1990).

\section{Approches chronologiques}

Une troisième conception du temps renvoie à une approche chronologique, privilégiant des systèmes de représentation articulant dimension quantitative et qualitative. Il peut s'agir par exemple de représenter à travers une série de dates et de noms, une succession d'étapes, subdivisées, allant d'un point d'origine jusqu'au temps présent. Ce type d'approche se retrouve évidemment dans les recherches en histoire (Pomian, 1984). On peut ainsi y avoir recours pour décrire l'évolution des pratiques éducatives. La notion de chronologie se retrouve également lorsqu'il s'agit de développer une forme spécifique de "conscience temporelle». Principalement développée dans l'enseignement de l'histoire et des langues, elle repose sur la transmission d'un vocabulaire lié au temps, et sur le développement 
de compétences linguistiques ou historiques fondées sur la capacité à représenter des séquences d'événements (Hoodless, 2002). Elle se retrouve également à chaque fois que l'enseignement d'une matière implique des aspects évolutifs, comme c'est par exemple le cas en biologie (Smith, 2010).

\section{Chronosophies}

Finalement, une quatrième manière de concevoir le temps en éducation ressort à travers la façon dont on interprète les relations entre passé, présent et futur, ce que Pomian (1984) nomme «chronosophie». Une chronosophie ne se contente pas de ce qui est perçu, observé, ou déduit des observations. Elle se légitime à partir du recours à diverses techniques visant à rendre l'avenir accessible, à en faire un objet de connaissance et de prédiction (Pomian, 1984, pp. v-vii). Ainsi, lorsqu'on envisage les recherches en éducation et les politiques qu'elles informent (Leaton Gray, 2004), tout discours produit sur l'éducation s'appuie - explicitement ou non - sur des chronosophies spécifiques. Celles-ci déterminent la manière dont chercheurs, apprenants, praticiens et décideurs, interprètent les relations de causes à effets entre des opportunités d'apprentissage ou de développement, passées, présentes et futures. Evoquer les notions de retard, de progrès, ou de développement supposent ainsi qu'on soit en mesure de retracer ou d'anticiper les étapes d'un processus de croissance. De même, dans la pratique pédagogique, dès lors qu'on mobilise les idées d'anticipation, d'orientation, de projet, ou de planification (Boutinet, 2008; Clegg, 2010), on introduit ce faisant une démarche d'apprentissage reposant sur différentes formes de chronosophie.

\section{Concevoir la pluralité des temporalités éducationnelles}

Reconnaître la pluralité des formes de temporalité impliquées en éducation rend nécessaire l'adoption d'une définition qui permette de rendre compte de leurs traits communs et de leur hétérogénéité. Comme le relève Pomian (1984), le temps en soi n'est jamais observable; ce que l'on peut observer ou étudier, ce sont les relations qui existent entre des changements coordonnés, dont le déroulement exprime une certaine forme de régularité. L'adoption d'une telle conception pluraliste comporte deux implications. La première est qu'elle permet d'envisager les pratiques éducationnelles comme productrices de temporalités, dans la mesure où elles coordonnent des changements qui s'opèrent de manière rythmée. Ainsi la structure cyclique de l'école (Ben-Peretz \& Bromme, 1990), ou les rythmes de l'alternance qui caractérisent l'éducation duale (Geay, 1999), participent à l'institutionnalisation de temporalités sociales distinctes. De même, le temps didactique qui rend compte de la succession de séquences d'essai-erreur à travers laquelle un apprenant développe une compétence spécifique dispose lui aussi de son organisation temporelle particulière (Chopin, 2010). C'est également le cas lorsqu'une personne rédige un récit de vie et qu'à travers la temporalité spécifique du processus d'écriture, elle recompose son rapport à l'existence 
(Dominicé, 1990). La seconde implication inhérente à une approche pluraliste du temps en éducation est qu'elle permet d'envisager les temporalités éducationnelles comme un tissu où s'enchevêtre une multitude de formes de changement (p.ex., physiques, biologiques, psychologiques, sociaux, culturels), disposant de leurs propres régularités et s'influençant mutuellement, de manières à la fois complémentaires, contradictoires et antagonistes.

\section{Concevoir le caractère hybride des temporalités éduca- tionnelles}

L'une des caractéristiques centrales des temporalités éducationnelles réside dans leur caractère hybride. Quelle que soit la nature des processus d'apprentissage, de transformation ou de développement auxquels on fait référence, tout phénomène éducationnel implique des temporalités hétérogènes qui sont fondamentalement enchevêtrées. Cela ressort par exemple à travers les enjeux inhérents à l'apprentissage d'une langue. Celle-ci repose sur une organisation du discours qui est le produit d'une histoire collective. Elle manifeste également une rythmicité qui est propre à l'organisation même du langage et qui caractérise les productions culturelles qui y sont associées (notamment en littérature). Lorsqu'on envisage son assimilation, celle-ci repose sur des processus neurologiques et cognitifs dont les temporalités varient notamment en fonction de l'âge biologique. De même, elle suppose le déroulement de relations interpersonnelles, elles-mêmes régulées en fonction de temporalités sociales (p.ex., la fréquence des interactions au sein d'une classe ou en famille). De telles interactions peuvent ou non être médiatisées par des supports matériels (p.ex., manuel de lecture) ou technologiques (p.ex., plateforme d'échanges) contraignant eux-aussi les temporalités inhérentes au transfert d'informations. Finalement, l'apprentissage d'une langue s'inscrit dans la vie d'une personne pour laquelle les significations et la valeur qui lui sont attribuées dépendent de sa propre biographie. Ainsi, les temporalités impliquées dans l'apprentissage d'une langue sont hybrides, car elles sont à la fois physiques, physiologiques, psychologiques, linguistiques, historiques, et sociales. Le temps de l'apprentissage d'une langue est ainsi à concevoir comme un composite hybride, fruit de l'enchevêtrement de changements qui s'opèrent de manière à la fois continue et discontinue à différents niveaux de l'expérience. La possibilité de reconnaître cette complexité est probablement l'une des caractéristiques fondamentales qui distingue et qui fait toute la valeur des savoirs produits en sciences de l'éducation (Charlot, 1995). Toutefois, la prise en considération de cette hybridité suppose d'interroger comment elle peut être étudiée, sans être réduite à l'une ou l'autre des temporalités impliquées dans l'apprentissage et le développement humain. Penser la question du temps en éducation, de manière non triviale, implique dès lors d'interroger notre capacité à penser la mise en lien et l'articulation entre des phénomènes de nature hétérogène, ce que Morin (1990) conçoit comme une intelligence de la complexité. 


\section{Deuxième axe de réflexion: envisager la nature dynamique et conflictuelle des temporalités éducationnelles}

La complexité et l'hétérogénéité des temporalités éducationnelles apparait de manière très concrète lorsqu'on s'intéresse aux tensions qui caractérisent leurs interrelations et leurs influences réciproques. Afin d'envisager la nature conflictuelle inhérente à l'expérience du temps et la façon dont elle détermine les processus éducatifs, deux postulats doivent être formulés. Le premier est qu'il est nécessaire de s'intéresser à la dimension dynamique qui caractérise les rapports entre des temporalités hétérogènes. L'influence des temporalités physiques, biologiques, psychologiques, sociales ou culturelles, impliquées dans les phénomènes éducatifs n'est en effet jamais constante; elle fluctue et elle évolue. Faire référence à l'idée de dynamique suppose ainsi de prendre en considération la présence de forces en interaction et en opposition. Dès lors qu'on reconnaît la présence de temporalités hétérogènes, on doit également admettre que leurs effets et leurs influences réciproques traduisent des rapports de force, voire des rapports de pouvoir. Le second postulat est qu'il est nécessaire de disposer d'une méthode pour envisager la complexité des dynamiques étudiées. Les filiations théoriques dans lesquelles s'inscrivent cette contribution privilégient ainsi une lecture multiréférentielle (Ardoino, 1999) qui suppose d'envisager les phénomènes éducatifs en les considérant simultanément à partir des logiques institutionnelles, organisationnelles, groupales, interpersonnelles et intrapsychiques qui les animent et les rendent conflictuels. Afin de problématiser l'expérience du temps dans une perspective à la fois dynamique et multiréférentielle, il apparaît particulièrement heuristique de faire référence à la notion de "contrainte temporelle» (Alhadeff-Jones, 2017).

\section{De la notion de contrainte temporelle}

La notion de "contrainte temporelle» renvoie à l'influence que peut avoir une temporalité donnée et, plus spécifiquement, le fait qu'elle peut conduire à confiner, limiter, restreindre ou mettre sous tension les opérations impliquées par un phénomène - tel que l'apprentissage, la transformation ou le développement individuel ou collectif - ainsi que les représentations que l'on s'en fait (Alhadeff-Jones, 2017, p. 52). Chacun fait au quotidien l'expérience récurrente de contraintes temporelles. Ainsi, des rythmes physiques tels que l'alternance entre le jour et la nuit, ou le rythme des saisons, déterminent le cadre dans lequel se déroule notre activité et l'expérience que l'on en a. De même, chacun sait combien il peut être difficile d'enseigner à certaines périodes de la journée, en raison de rythmes biologiques tels que ceux du sommeil ou de la digestion. Nos états psychologiques, notre capacité d'attention, et nos humeurs ont également leur propre rythmicité. De même, lorsque des contenus sont transmis, ils impliquent également des contraintes temporelles liées à leur envergure (p.ex., 
la longueur d'un texte) ou au moyen de communication utilisé (p.ex., écrit ou oral, synchrone ou asynchrone). De plus, chacun est contraint par les rythmes sociaux qui régulent nos interactions et se manifestent à travers les routines, les programmes, ou les calendriers auxquels nous nous soumettons, jour après jour. Il est également important de souligner le fait qu'une contrainte temporelle peut s'exercer à la fois de manière fonctionnelle sur le déroulement d'une activité, et de manière symbolique, sur la manière dont on se représente les changements qui nous affectent. Ainsi, l'adoption d'un horaire de travail contraint l'activité, au même titre que l'imaginaire dominant d'un temps abstrait, mesurable et quantifiable, envisagé comme une ressource monnayable conditionne et limite de manière significative notre compréhension du temps (Ardoino, 2000; Castoriadis, 1975).

\section{L'évolution des contraintes temporelles comme analyseur de l'évolution de l'éducation}

L'évolution des contraintes temporelles constitue un analyseur particulièrement significatif pour rendre compte de l'évolution sociale, culturelle et politique des pratiques et des théories éducatives. En effet, l'histoire de l'éducation est caractérisée par l'émergence de stratégies développées au fil des siècles pour maîtriser des contraintes temporelles, à la fois universelles et contingentes (p.ex., physiologiques, cognitives, discursives, environnementales ou sociales) (Alhadeff-Jones, 2017). Sur le plan institutionnel, par exemple, les temporalités de l'instruction et de la formation ont été au cours de leur histoire influencées et contraintes par les rythmes animant des institutions telles que le pouvoir politique, le travail, la famille, la religion, le commerce, l'industrie, et les loisirs. En éducation, ces contraintes temporelles s'exercent autant sur le déroulement des activités que sur le plan symbolique. Ainsi, l'imaginaire occidental contemporain du temps scolaire est encore conditionné par un héritage qui remonte aux pratiques monastiques et à la Révolution industrielle, privilégiant une organisation des rythmes vécus favorisant l'obéissance et l'efficience (St-Jarre \& Dupuy-Walker, 2001; Jeanmart, 2007). L'histoire de l'éducation témoigne aussi du fait que les pratiques et les discours éducatifs produisent des formes spécifiques de contraintes temporelles s'exerçant sur les corps, sur les discours et sur les modalités d'interaction sociale. Ainsi, depuis toujours, les pratiques éducatives s'appuient sur l'exercice d'une "discipline temporelle», fondée sur l'imposition de rythmes qui contraignent la manière de s'exprimer, de se mouvoir et d'interagir en groupe et au sein de la société, en fonction de règles qui varient selon les époques et les cultures (Foucault, 1975). De même, les pratiques et les théories éducatives alimentent, autant qu'elles limitent, un imaginaire à travers lequel on se représente les temporalités de l'apprentissage, du changement et du développement, notamment en regard des âges de la vie. 
Troisième axe de réflexion: envisager la portée critique d'une réflexion sur la complexité temporelle en éducation

L'expérience de contraintes temporelles est indissociable des enjeux éthiques et critiques qu'elle soulève. En sciences de l'éducation, ils ressortent par exemple lorsqu'on interroge les conditions nécessaires à la poursuite d'un projet émancipateur, renvoyant aux apprentissages à partir desquels individus et collectifs sont en mesure de maintenir ou d'accroître leur liberté de pensée, leur autonomie et leur pouvoir d'agir. Dans le contexte sociétal contemporain, interroger la nature des rapports entre émancipation et expérience temporelle constitue un axe de réflexion particulièrement crucial. Pour l'appréhender, il convient dans un premier temps d'évoquer certaines des formes de contrainte temporelle qui se sont renforcées en éducation au fil du $20^{\mathrm{e}}$ siècle, et de dégager en quoi elles peuvent être vécues comme aliénantes. Ce faisant, il est possible de mettre en évidence le fait qu'une réflexion sur le temps en éducation comporte une portée critique inhérente à la capacité de rendre intelligible et de mettre en cohérence, non seulement sur le plan intellectuel, mais aussi et surtout sur le plan existentiel, les rapports complémentaires, contradictoires et antagonistes entre les différentes temporalités qui rythment nos vies et notre éducation.

\section{L'expérience du temps comme source de souffrance}

En tant qu'apprenants, citoyens, professionnels, praticiens, ou chercheurs, beaucoup d'entre nous sont aujourd'hui affectés par l'évolution de "cadres temporels» (Grossin, 1996) au sein desquels chacun est en prise avec «le» temps, et au sein de laquelle il semble de plus en plus difficile d'entretenir une capacité d'agir. Soit que l'on ne trouve pas le temps de «ralentir» pour souffler, prendre du recul, ou simplement pour lire ou réfléchir. Soit que l'on se sente enfermés dans un carcan temporel duquel on n'arrive pas à s'extraire. A bien des égards l'expérience du temps apparaît désormais comme une source de souffrance. L'un des symptômes en est l'épuisement professionnel: rupture qui émerge à partir de la contrainte exercée par les temporalités du travail sur les temporalités de notre organisme et de notre psychisme. À l'inverse, l'absence de travail peut également être vécue par ceux qui n'ont pas d'emploi comme une source d'«exil temporel» (Lesourd, 2006); la souffrance vient alors de la difficulté à rythmer l'activité quotidienne, en l'absence d'un temps social vécu comme structurant. Bien qu'elles ne soient pas nouvelles, la plupart des formes de contraintes temporelles qui s'exercent aujourd'hui, sur et à travers les pratiques éducatives, semblent s'être accentuées (Alhadeff-Jones, 2017). Pour le comprendre, trois phénomènes centraux qui se sont renforcés dans le courant du $20^{\mathrm{e}}$ siècle peuvent être évoqués: l'internalisation de double contraintes temporelles, la reconnaissance de la nature discontinue des processus éducatifs, et l'accroissement d'un sentiment d'accélération et d'urgence. 


\section{Le vécu de double contraintes temporelles}

Comme l'évoque Perrenoud (2001), l'école moderne enferme les enseignants dans un dilemme permanent entre d'une part l'efficience requise par un système basé sur un usage rationnel du temps d'enseignement, et d'autre part, un idéal d'équité qui implique de reconnaître et de respecter les rythmes singuliers de chaque élève, y compris ses rythmes biologiques. Ainsi, l'expérience de ce type de dilemme peut apparaître comme une source de souffrance lorsque ces deux impératifs ne sont plus compatibles et qu'il n'est pas possible de s'extraire de cette contradiction. Lorsqu'elle ne peut pas être explicitée et qu'il n'est pas possible d'agir à son encontre, cette expérience peut être vécue comme une «double contrainte» aliénante (Bateson, Jackson, Haley \& Weakland, 1956). Dans la mesure où elle est présentée comme un allant de soi, et qu'elle est produite au sein d'un système qu'il est difficile de remettre en question, l'expérience de «double contraintes temporelles» peut être internalisée, et conduire dès lors à adopter des comportements dysfonctionnels (Alhadeff-Jones, 2017).

\section{Ruptures et discontinuités dans les processus éducatifs et de formation}

Une autre source de tensions apparaît à travers la place croissante accordée à l'expérience de ruptures et de discontinuités dans les processus éducatifs et de formation. Celles-ci peuvent être envisagées au cœur de l'activité d'apprentissage (Whitehead, 1929/1967), ou au sein de dispositifs de formation telle que l'alternance (Geay, 1999), privilégiant une forme de rythmicité. Ruptures et discontinuités occupent également une place centrale dans la problématique du «tierstemps scolaire» et de la mise en cohérence entre éducation formelle et informelle (Sue \& Rondel, 2001). Elles sont aussi au centre des discours sur l'apprentissage tout au long de la vie qui conferent à l'idéal d'éducation permanente (Pineau, 2000) la fonction de relier les apprentissages discontinus qui ponctuent l'existence, notamment en raison des reconfigurations de trajectoires professionnelles. En formation d'adultes, l'expérience vécue d'épiphanies, de crises ou d'épreuves, renvoient finalement aux discontinuités à travers lesquelles se réorganisent et se transforment les significations conférées aux apprentissages de la vie sur le plan existentiel (Alhadeff-Jones, 2017, à paraître). L'expérience de discontinuités et de ruptures apparaît ainsi comme une forme de contrainte temporelle prépondérante en éducation qui, lorsqu'elle peut être intégrée et élaborée, se révèle comme un moyen puissant d'apprentissage et de développement. Toutefois, lorsque ce n'est pas le cas, elle peut contribuer à un sentiment d'absence de cohérence, de désorientation, et d'aliénation, inhérent à l'incapacité d'attribuer un sens constructif à une expérience vécue comme fragmentée, divisée ou compartimentée. 
L'impact des phénomènes d'accélération sur la société Depuis une trentaine d'année, la prépondérance de la vitesse avec laquelle les progrès technologiques et les rapports sociaux évoluent constitue une source croissante de préoccupation. La diffusion de l'urgence comme nouvelle norme (Bouton, 2013) et l'identification de phénomènes d'accélération (Rosa, 2010) renforcent ainsi un sentiment diffus de pression temporelle. De tels phénomènes en soi ne sont pas nouveaux. On retrouve au début du $20^{\mathrm{e}}$ siècle l'expression d'un malaise similaire, conséquence des transformations technologiques, culturelles et sociales, associées aux effets de la Révolution industrielle et à l'émergence d'une société capitaliste globalisée. Dans le contexte contemporain, ces transformations nous affectent d'autant plus qu'elles tendent à accentuer les effets inhérents aux contraintes temporelles évoquées précédemment. Ainsi, la valorisation de la vitesse et de l'accélération dans les différentes sphères de l'existence s'ajoute aux contradictions vécues lorsqu'il s'agit de trouver un équilibre entre les différents rythmes de nos activités et de les mettre en cohérence. En éducation, l'introduction de formations «accélérées» (Wlodkowski, 2003) met ainsi en évidence certaines des ambivalences inhérentes à la compression et à la standardisation des programmes, susceptibles de contribuer à un sentiment de fragmentation et de superficialité. Sur le plan professionnel et existentiel, l'accroissement du rythme de certaines activités contribue également à renforcer l'omniprésence de ce que Pineau (2000) nomme la «schizochronie»: le vécu de temporalités divisées et clivées les unes des autres qui participe à un sentiment de perte de soi et d'aliénation.

\section{L'aliénation temporelle comme rupture entre l'expérience du temps et les significations qui lui sont attribuées}

L'une des caractéristiques communes que l'on retrouve chez les auteurs qui font référence à l'idée d' "aliénation temporelle» ressort à travers la coupure, la distorsion (Rosa, 2010), ou le découplage (Thompson, 1967) que l'on peut observer entre deux expériences du temps. Pour Castoriadis (1975), le sentiment d'aliénation associé à l'expérience du temps vient du fait que l'expérience vécue est dominée par des conceptions abstraites ou réifiées du temps, à partir desquelles nos activités sont régulées. D'un côté, on évoque une expérience «qualitative» du temps, perçu comme signifiant; le temps vécu est en cohérence avec la manière dont on attribue du sens à l'expérience quotidienne et la durée dans laquelle elle s'inscrit. D'autre part, on est confronté à une conception du temps strictement quantitative, standardisée et abstraite. Celle-ci s'impose à nous dans le cadre de nos activités, notamment via les dynamiques sociales qui cadrent l'éducation formelle ou le contexte de travail. L'expérience du temps devient un enjeu de calcul, de rentabilité et surtout d'efficience, indépendant de la qualité de l'expérience vécue. 


\section{Apprendre à développer son rapport au temps et aux}

rythmes vécus: un enjeu critique de formation

À travers ces considérations, ce qui apparaît aujourd'hui comme critique c'est le développement d'une capacité à mettre en cohérence et à organiser des expériences hétérogènes du temps, telles qu'on peut être amené à les vivre au cours des différents moments de notre existence: à l'école, dans les loisirs, au travail, en famille, dans le monde économique, dans la cité, dans les arts, etc. (Pineau, 2000). Le terme d'organisation n'est pas envisagé ici à partir d'une conception instrumentale, telle qu'on la retrouve dans les démarches de «gestion du temps», qui supposerait la compétence à organiser ses activités en fonction d'un emploi du temps rationnel et étalonné. De même, elle ne relève pas juste d'un aménagement ou de la planification de l'activité, comme c'est le cas dans les travaux sur les «temporalités de l'enseignement» (Chopin, 2010; Delhaxhe, 1997) ou sur les «rythmes scolaires» (Cavet, 2011) qui se limitent à la prise en considération de temporalités et de rythmes objectivables, mesurables et quantifiables. Le terme d'organisation renvoie davantage à la capacité d'articuler des significations hétérogènes associées à nos expériences. Elle renvoie au fait que les rythmes qui façonnent les différentes activités de nos vies, et les différentes contraintes temporelles qui y sont associées, sont porteurs de significations du temps qui se révèlent à la fois complémentaires, contradictoires et antagonistes. Il s'agit fondamentalement d'apprendre à se les représenter et à les relier. Pour ce faire, on doit être en mesure d'identifier les rythmes et les temporalités en présence, afin de privilégier ceux qui permettent de majorer l'apprentissage, les processus d'individuation (Michon, 2005), de développement et d'émancipation. On doit également pouvoir interroger la façon dont on interprète le sens et la valeur qu'ils prennent. Il s'agit finalement d'être capable de comprendre les manières dont ils interagissent et s'influencent mutuellement dans le cours de nos vies (Alhadeff-Jones, 2014, 2017).

\section{Ouvertures}

L'objectif de ce texte était d'interroger certains des présupposés à partir desquels les temporalités de l'éducation et de la formation peuvent être envisagées dans leur complexité. Pour ce faire, trois axes de réflexion ont été proposés. Le premier axe a conduit à formuler l'une des spécificités inhérentes à une réflexion sur le temps en éducation. Alors que les conceptions du temps véhiculées en philosophie, en physique, en biologie ou en sciences humaines tendent à réduire la complexité temporelle des phénomènes humains en fonction de logiques disciplinaires, la recherche en sciences de l'éducation ne peut faire l'économie d'une réflexion visant à articuler les différents registres physiques, biologiques, psychologiques, et sociaux de l'expérience. Toute pratique éducative et tout processus de formation impliquent en effet une hybridité temporelle caractérisée par des rythmes hétérogènes (p.ex., langagiers, corporels, sociaux) qui s'influencent mutuellement. L'enjeu pour les chercheurs en sciences de l'éducation serait ainsi 
d'en penser les conditions d'articulation, autant sur le plan épistémologique que sur le plan de l'expérience, afin d'envisager des formes d'organisation temporelle propices à majorer l'apprentissage et le développement. Le deuxième axe a exploré la dimension contraignante inhérente à l'expérience du temps, autant sur le plan fonctionnel de l'activité que sur le plan symbolique des représentations. À partir de la notion de "contrainte temporelle», il a été proposé d'envisager l'évolution des pratiques éducatives à la fois à partir des rythmes qui les déterminent et les mettent sous tension, et de ceux qu'elles imposent à l'activité humaine, autant sur le plan individuel que collectif. Interroger l'hétérogénéité des contraintes temporelles vécues se révèle ainsi un ressort puissant pour repenser et enrichir l'exercice éducatif et les processus de formation. Le troisième axe de réflexion a interrogé plus spécifiquement certaines des contraintes temporelles inhérentes à l'environnement sociétal contemporain (vécu de doubles contraintes, de ruptures et de discontinuités, exacerbé par un sentiment d'urgence et d'accélération). Il suggère finalement de revisiter l'idéal d'émancipation associé à une éducation humaniste en interrogeant sa dimension rythmique. Et si la finalité d'une éducation critique était d'apprendre à identifier les «rythmes justes» dans un contexte ou à un moment donné? Au-delà d'une rhétorique de la vitesse ou de la lenteur, l'enjeu fondamental serait ainsi de disposer d'un langage et d'une capacité à discriminer, interpréter, argumenter, évaluer, juger et remettre en question l'éventail temporel des rythmes qui façonnent les activités à travers lesquels individus et collectivités apprennent et se forment tout au long de leur existence.

\section{Note}

1 Cet article présente une version éditée du texte de la conférence introductive - intitulée "Revisiter les rythmes de l'éducation et de la formation dans une perspective critique» - que j'ai présentée lors du congrès annuel 2017 de la Société Suisse pour la Recherche en Education, le 26 juin 2017 à l'Université de Fribourg.

\section{Références}

Adam, B. (1994). Time and social theory. Cambridge: Polity Press.

Alhadeff-Jones, M. (2007). Education, critique et complexité. Lille, France: Université de Lille, Atelier National de Reproduction des Thèses.

Alhadeff-Jones, M. (2010). Challenging the limits of critique in education through Morin's paradigm of complexity. Studies in Philosophy and Education, 29, (5), 477-490.

Alhadeff-Jones, M. (2014). Pour une approche réflexive et critique des rapports entre temporalités et professionnalisation. Revue Phronesis, 3, (4), 4-12.

Alhadeff-Jones, M. (2017). Time and the rhythms of emancipatory education. Rethinking the temporal complexity of self and society. London: Routledge.

Alhadeff-Jones, M. (à paraître). Pour une conception rythmique des apprentissages transformateurs. Revue Phronesis.

Ardoino, J. (1999). Education et politique. Paris: Anthropos.

Ardoino, J. (2000). Les avatars de l'éducation. Paris: Presses Universitaires de France.

Bateson, G., Jackson, D. D., Haley, J. \& Weakland, J. (1956). Toward a theory of schizophrenia. Behavioral Science, 1, (4), 251-254. 
Ben-Peretz, M. \& Bromme, R. (Éd.). (1990). The nature of time in schools: Theoretical concepts, practitioner perceptions. New York, NY: Teachers College, Columbia University.

Boutinet, J. P. (2008). Penser l'anticipation dans sa (ses) crise(s). Education Permanente, 176, $8-22$.

Bouton, C. (2013). Le temps de l'urgence. Lormont, France: Le Bord de l'eau.

Castoriadis, C. (1975). L'institution imaginaire de la société. Paris: Seuil.

Cavet, A. (Éd.). (2011). Rythmes scolaires: Pour une dynamique nouvelle des temps éducatifs. Dossier d'actualité de l'Institut National de Recherche Pédagogique, 60.

Charlot, B. (1995). Les sciences de l'éducation: Un enjeu, un défi. Paris: ESF.

Chenet, F. (2000). Le temps: Temps cosmique, temps vécu. Paris: Armand Colin.

Chopin, M.-P. (2010). Les usages du 'temps' dans les recherches sur l'enseignement. Revue Française de Pédagogie, 170, 87-110.

Clegg, S. (2010). Time future: The dominant discourse of higher education. Time \& Society, 19, (3), 345-364.

Delhaxhe, A. (1997). Le temps comme unité d'analyse dans la recherche sur l'enseignement. Revue Française de Pédagogie, 118, 107-125.

Dominicé, P. (1990). L'histoire de vie comme processus de formation. Paris: L'Harmattan.

Dubar, C. (2008). Temporalité, temporalités: Philosophie et sciences sociales. Temporalités. Revue de sciences sociales et humaines, 8. Consulté le 5 mai 2014 dans http://temporalites.revues.org/137.

Foucault, M. (1975). Surveiller et punir. Paris: Gallimard.

Fraisse, P. (1957). Psychologie du temps. Paris: Presses Universitaires de France.

Fraser, J. T. (Éd.). (1966). The voices of time. New York, NY: George Braziller.

Geay, A. (1999). Actualité de l'alternance (note de synthèse). Revue Française de Pédagogie, $128,107-125$.

Gonord, A. (2001). Le temps. Paris: Flammarion.

Grossin, W. (1996). Pour une science des temps. Toulouse, France: Octares.

Hoodless, P. A. (2002). An investigation into children's developing awareness of time and chronology in story. Journal of Curriculum Studies, 34, (2), 173-200.

Jeanmart, G. (2007). Généalogie de la docilité dans l'Antiquité et le Haut Moyen Âge. Paris: Vrin.

Koukkari, W. L. \& Sothern, R. B. (2006). Introducing biological rhythms. New York, NY: Springer.

Larsson, J. \& Sanne, C. (2005). Self-help books on avoiding time shortage. Time \& Society, 14, (2/3), 213-230.

Leaton Gray, S. (2004). Defining the future: An interrogation of education and time. British Journal of Sociology of Education, 25, (3), 323-340.

Lesourd, F. (2006). Des temporalités éducatives: Note de synthèse. Pratiques de Formation / Analyses, 51-52, 9-7.

Lurçat, F. (1995). Le temps de la physique. L'enseignement philosophique, 46, (1), 7-33.

Mayes, C. (2005). Teaching and time: Foundations of a temporal pedagogy. Teacher Education Quarterly, 32, (2), 143-160.

Michon, P. (2005). Rythmes, pouvoir, mondialisation. Paris: Presses Universitaires de France.

Morin, E. (1990). Introduction à la pensée complexe. Paris: Seuil.

Perrenoud, P. (2001). Gérer le temps qui reste: L'organisation du travail scolaire entre persécution et attentisme. In C. St-Jarre \& L. Dupuy-Walker (Éd.), Le temps en éducation: Regards multiples (pp. 287-316). Sainte-Foy, QC: Presses de l'Université du Québec.

Pineau, G. (2000). Temporalités en formation: Vers de nouveaux synchroniseurs. Paris: Anthropos.

Pomian, K. (1984). L'ordre du temps. Paris: Gallimard.

Rosa, H. (2010). Accélération. Une critique sociale du temps (D. Renault, trad.). Paris: La Découverte. 
Slattery, P. (1995). A postmodern vision of time and learning: A response to the National Education Commission Report 'Prisoners of time'. Harvard Educational Review, 65, (4), 612-633.

Smith, M. U. (2010). Current status of research in teaching and learning evolution. Science \& Education, 19, (6-8), 523-571.

St-Jarre, C. \& Dupuy-Walker, L. (Éd.). (2001). Le temps en éducation: Regards multiples. Sainte-Foy, QC, Canada: Presses Universitaires du Québec.

Sue, R. \& Rondel, Y. (2001). Rythmes de vie et éducation: Education et modes de vie. Les Cahiers Millénaire 3, (24), 25-53.

Testu, F. (2008). Rythmes de vie et rythmes scolaires. Issy-les-Moulineaux, France: Masson.

Thompson, E. P. (1967). Time, work-discipline and industrial capitalism. Past and Present, $38,56-97$.

Whitehead, A. N. (1929/1967). The aims of education and other essays. New York, NY: The Free Press.

Wlodkowski, R. J. (2003). Accelerated learning in colleges and universities. New Directions for Adult and Continuing Education, 97, 93-97.

Mots-clés: Temps, rythme, complexité, éducation, apprentissage, temporalités éducatives

\section{Zeitliche Komplexität in der Bildung aus einer kritischen Perspektive begreifen}

\section{Zusammenfassung}

Die Untersuchung von Zeit und Rhythmen in Erziehungsprozessen weist eine Komplexität auf, die besondere schwer zu analysieren ist, weil sie gleichzeitig auf heterogene Aspekte der Erfahrung (z.B. physische, biologische, psychologische, soziale, kulturelle) Bezug nehmen muss. Um diese Komplexität verständlich zu machen, wird vorgeschlagen, einige Annahmen über die Zeitlichkeit in der Erziehung und Bildung zu hinterfragen. Die Betrachtung gliedert sich anhand von drei Achsen: Die erste zielt auf eine theoretische Rahmung der Heterogenität zeitlicher Aspekte von Erziehung und Bildung; die zweite beleuchtet deren Dynamik und Widersprüchlichkeit; die dritte hinterfragt aus einer emanzipatorischen Perspektive kritisch das Ziel der Reflexion zeitlicher Komplexität von Bildung.

Schlagworte: Zeit, Rhythmus, Komplexität, Bildung, lernen, zeitliche Aspekte von Bildung 


\section{Una prospettiva critica per concepire la complessità temporale in educazione}

\section{Riassunto}

Lo studio del tempo e dei ritmi dell'educazione rivela una complessità difficile da analizzare, poiché si riferisce simultaneamente ad aspetti eterogenei dell'esperienza (per esempio a quello fisico, biologico, psicologico, sociale e culturale). Per poter comprendere tale complessità, il presente articolo si propone di interrogare i presupposti attraverso i quali vengono concepite le differenti temporalità dell'educazione. Questa riflessione è articolata secondo tre assi: il primo ha come scopo di inquadrare/incorniciare l'eterogeneità delle temporalità educative, il secondo esplora la loro natura dinamica e conflittuale; il terzo asse interroga, in modo più specifico, l'intento critico di una riflessione sulla complessità temporale, concepita dalla prospettiva di un'educazione emancipatoria.

Parole chiave: Tempo, ritmo, complessità, educazione, apprendimento, temporalità educativa

\section{Conceiving temporal complexity in education from a critical perspective}

\section{Summary}

The study of time and the rhythms of education reveals a complexity that is particularly difficult to analyze, because it refers simultaneously to heterogeneous aspects of experience (e.g., physical, biological, psychological, social, cultural). To make such a complexity understandable, this paper question some of the assumptions through which the temporalities of education and formation are conceived. This reflection is organized around three axes: the first one aims at framing the heterogeneity of educational temporalities; the second one explores their dynamic and conflicting nature; and the third axis questions more specifically the critical aim of a reflection on temporal complexity, conceived from the perspective of emancipatory education.

Keywords: Time, rhythm, complexity, education, learning, educational temporalities 